\title{
ANDES

www.scielo.cl

\section{Efectos del confinamiento por COVID-19 en la demanda de atención de niñas, niños y adolescentes con quemaduras}

\author{
Effects of COVID-19 lockdown on the demand for health care of children and \\ adolescents with burns
}

\author{
Fresia Solís F.a, Rolando Saavedra O. ${ }^{\mathrm{b}}$, Karina Zalavari P.,
}

aDirección de Extensión, Docencia e Investigación, COANIQUEM. Santiago, Chile

bDirección médica, COANIQUEM. Santiago, Chile

cEnfermera

Recibido: 13 de enero de 2021; Aceptado: 11 de mayo de 2021

\section{¿Qué se sabe del tema que trata este estudio?}

En tiempo de pandemia COVID-19, los estudios encontrados de demanda de atención de niños con quemaduras, son europeos, en pequeñas muestras y referidos principalmente a hospitalizados. En pacientes ambulatorios, los resultados son contradictorios respecto de la demanda de atención.
¿Qué aporta este estudio a lo ya conocido?

En fase inicial de la pandemia por COVID-19, con confinamiento estricto, se produjo una disminución de la demanda de atención ambulatoria por lesiones de quemadura, afectando por igual a pacientes con lesiones agudas y con requerimiento de rehabilitación de secuelas.

\section{Resumen}

La pandemia de COVID-19 ha implicado adaptarse a una realidad diferente, con largos confinamientos intradomiciliarios, lo que podría favorecer un aumento de quemaduras infantiles en el hogar. Objetivo: Comparar la situación epidemiológica de pacientes que ingresaron a la Corporación de Ayuda al Niño Quemado (COANIQUEM) por lesiones de quemaduras de manejo ambulatorio al inicio del confinamiento por COVID-19 en contraste con el año anterior. Pacientes y Método: Estudio analítico y transversal. La población correspondió a 2.027 pacientes menores de 20 años que ingresaron por primera vez en alguno de los Centros de COANIQUEM solo por lesiones de quemaduras, entre abril y julio de 2019 y 2020. Se recolectó el número de pacientes atendidos en cada mes y variables demográficas, sociales y clínicas. Se calculó el porcentaje de cambio mensual comparando las características de los pacientes de ambos años. Resultados: Durante 2020, se observó una disminución del 48,7\% de los ingresos ambulatorios totales. Hubo un aumento relativo del 10,5\% de las quemaduras en menores de 5 años, del $18,3 \%$ de aquellas ocasionadas por líquido caliente, del 33,1\% del número de

Palabras clave: Quemaduras; COVID-19;

Niños;

Adolescentes

Correspondencia:

Fresia Solís F.

fresiasolis@hotmail.com 
lesiones en 3 o más localizaciones y del 16,8\% de las quemaduras que ocurrían en el hogar propio. No influyó ubicación geográfica, sexo ni nivel socioeconómico. Conclusiones: En el período inicial de la pandemia por COVID-19, con confinamiento estricto, se produjo una disminución de la demanda de atención por lesiones de quemadura, afectando por igual a pacientes ambulatorios con lesiones agudas, como para rehabilitación de secuelas, secundario a la disminución efectiva de la ocurrencia de quemaduras y a la menor posibilidad de recurrir a atención de salud.

\begin{abstract}
COVID-19 pandemic has meant adapting to a different reality, with long-term lockdowns that might cause an increase of burns in children at home. Objective: To compare the epidemiological situation of patients admitted to the Corporación de Ayuda al Niño Quemado (COANIQUEM) due to outpatient burn injuries management at the beginning of COVID-19 lockdown with the same period the year before. Patients and Methods: Analytical and cross-sectional study. A population of 2,027 patients under the age of 20, who were admitted to COANIQUEM for the first time with burn injuries, between April and July of 2019 and 2020 was analyzed. The number of patients admitted each month was registered as well as their demographic, social, and clinical characteristics. The monthly percentage variation was calculated by comparing patient data in both years. Results: During 2020, there was a $48.7 \%$ decrease in overall outpatient admissions. There was a relative increase of $10.5 \%$ in burns in patients under 5 years old, $18.3 \%$ in scalds, $33.1 \%$ in the number of burns in 3 or more body locations, and $16.8 \%$ in burns occurring at home. These parameters were not influenced by geographic location, sex, or socioeconomic level. Conclusions: In the first period of the COVID-19 pandemic, with strict lockdown strategies, there was a decrease in the demand for burn care, affecting both outpatients with acute burns and those who were admitted for sequelae rehabilitation, as a result of the effective decrease in the burns incidence and the reduced access to health care.
\end{abstract}

Keywords: Burns; COVID-19; Children; Teenagers

\section{Introducción}

La pandemia por COVID-19 ha significado cambios en la vida cotidiana de la sociedad, afectando a todos los sectores, viéndose obligados a limitar aforos, restringir los servicios y adaptar la atención al público a esta nueva realidad. También ha afectado a los prestadores de salud, quienes han debido acomodarse a las normas nacionales e internacionales para manejar la emergencia.

En Chile, el primer caso confirmado de COVID-19 se registró el 3 de marzo de $2020^{1}$. A partir de este caso, el brote se expandió en el territorio nacional hasta alcanzar la totalidad de las regiones del país ${ }^{2}$.

El 18 de marzo de 2020, el Presidente de la República decretó el «estado [de excepción constitucional] de catástrofe» por 90 días $^{3}$. El 15 de junio, y mediante el decreto supremo $\mathrm{N}^{\circ} 104$ de 2020 del Ministerio del Interior y Seguridad Pública, se prorrogó dicho estado por 90 días más ${ }^{4}$. El 22 de marzo de 2020, se anunció un toque de queda para restringir el movimiento de personas a partir de dicho día -entre las 22:00 y las 05:00 horas- en todo el territorio nacional, sin un plazo de término y manteniendo operativas las líneas de abastecimientos, área de salud y emergencias ${ }^{5,6}$. Las clases de los niveles preescolar y escolar de forma presencial se suspendieron totalmente a comienzos de abril ${ }^{7-9}$.

El gobierno, a través del Ministerio de Salud (MIN-
SAL), ha tenido como uno de sus objetivos prevenir la propagación de la enfermedad instando a la población a quedarse en casa, practicar el lavado de manos, distanciamiento físico, el uso obligatorio de mascarilla, la cuarentena de los casos infectados, la restricción de viajes, el cierre de establecimientos, la cancelación de eventos masivos, la cuarentena por regiones y el confinamiento en general.

Los casos notificados como contagiados por COVID-19 aumentaron en el país inicialmente en forma lenta para elevarse sostenidamente entre los meses de mayo y julio con un máximo en junio de $2020^{10}$.

La pandemia COVID-19 también se ha traducido en un cambio de los estilos de vida, referente a la actividad laboral, educacional, familiar y social dentro del hogar. En estudios anteriores, sin pandemia, se ha observado que el $88 \%$ de las quemaduras en niños ocurrían en el hogar ${ }^{11,12}$ y que éstas aumentaban en los meses más fríos al estar en su interior ${ }^{12}$. Por estos motivos, podría esperarse que a mayor permanencia de los niños en las casas, la demanda de atención por quemaduras en especial en otoño e invierno aumentaría. Lo anterior, llevó a la Corporación de Ayuda al Niño Quemado (COANIQUEM) a realizar una intensa campaña de prevención de quemaduras por redes sociales para advertir a la población de los mecanismos más frecuentes que provocan estas lesiones. 
Diversos centros de atención de pacientes con quemaduras a nivel mundial, en un tiempo acotado al inicio de la pandemia, han dado a conocer su experiencia en relación al funcionamiento y manejo ${ }^{13-19}$, cambios epidemiológicos $^{20,21}$, llamados a la comunidad ${ }^{22,23} \mathrm{o}$ estudios sobre consultas, hospitalizaciones y duración del período de estadía hospitalaria ${ }^{24}$.

Los centros de rehabilitación de COANIQUEM ubicados en Antofagasta (Zona norte), Santiago (Zona centro) y Puerto Montt (Zona sur) mediante un convenio asistencial con MINSAL son establecimientos de referencia de la red pública, para la atención ambulatoria de niños, niñas y adolescentes (NNA) con lesiones de quemaduras, ya sea en etapa aguda por consulta espontánea, por derivación desde servicios de urgencia o al alta hospitalaria de varios establecimientos en todo el país para iniciar la rehabilitación de sus secuelas ${ }^{25}$.

Siguiendo las directrices ministeriales de prevención del COVID 19, los tres establecimientos mantuvieron la atención presencial a la población que solicitaba una asistencia de salud inicial, postergando controles que no fueran indispensables y efectuando tele-consulta a pacientes en etapa de seguimiento de su tratamiento por secuelas.

El objetivo de este estudio es dar a conocer la experiencia interna de atención de COANIQUEM y la situación epidemiológica de la población de NNA en el primer período de mayor gravedad de la pandemia COVID-19, en un análisis comparativo con el mismo período del año anterior.

\section{Pacientes y Método}

Estudio analítico y transversal. Se trabajó con la base de datos del Sistema Informático de la Institución (INFOQUEM), obteniéndose autorización del Comité Ético Científico de COANIQUEM para procesar la base con datos personales encriptados de los pacientes. (Certificado CEC_06-08-2020)

La población estuvo constituida por niños, niñas y adolescentes menores de 20 años, atendidos por primera vez en COANIQUEM en sus Centros de Antofagasta, Santiago y Puerto Montt, entre el $1^{\circ}$ de abril y el 31 de julio de los años 2019 y 2020, consultando en el "Policlínico de curaciones" por quemaduras en etapa aguda provocadas por cualquier agente causal o ingresados para rehabilitación en el "Policlínico de secuelas". Se excluyeron NNA con secuelas de otro origen (mordedura de perros, trauma o herida postquirúrgica).

Se recolectó el número de pacientes atendidos en cada mes en los Centros mencionados anteriormente identificando en especial los residentes en la comuna de Pudahuel donde se encuentra el Centro de Rehabilitación Santiago. Se registraron variables demográficas: género, edad y zona de procedencia; sociales: previsión en salud, como indicador de nivel socioeconómico; centro de atención, donde ocurrió la lesión, con quien vive el menor; y clínicas: agente causal, profundidad de la quemadura, localización, número de localizaciones, hora de la lesión y tratamiento en lugar de ocurrencia. Se calculó el porcentaje de cambio del número de pacientes atendidos en COANIQUEM y la comuna de Pudahuel, donde se ubica la institución en los meses de abril a julio, comparando año 2019 con 2020. Así mismo, se comparó el año 2020 con los 3 años anteriores para evaluar posibles variaciones debido a cambios climáticos. Las características demográficas, sociales y clínicas de los años seleccionados se incluyeron en planilla Excel, siendo procesadas con SPSS versión 25. Se obtuvieron pruebas de independencia basadas en distribución Chi cuadrado para las variables estudiadas. Todas las pruebas con $\mathrm{p}<0,05$.

\section{Resultados}

De la base de datos constituida por 2.315 pacientes atendidos en los tres centros ambulatorios en el período estudiado del año 2019 y 2020, se eliminaron 241 $(10,4 \%)$ por corresponder a pacientes atendidos por cicatrices de otro origen (mordedura de perro, trauma o herida post-quirúrgica). También, se excluyeron 47 pacientes (2\%) mayores de 20 años.

El porcentaje de cambio global en el número de pacientes atendidos en los meses estudiados entre los años 2019 y 2020, fue una disminución de $48,7 \%$ en el año de pandemia, y en comparación con 2017-2019 una disminución de 49,4\%. La mayor reducción de $64,3 \%$, se observó en el mes de mayo respecto del año 2019 y $65,3 \%$ en comparación con el trienio. Los ingresos de residentes en comuna de Pudahuel en el total disminuyeron $52,5 \%$ en los años comparados y $81,5 \%$ en el mes de mayo (tabla 1).

Entre las características demográficas y sociales, resultaron significativos los cambios en: edad ( $\mathrm{p} \leq 0,0263$ ), aumentando el porcentaje de niños menores de 5 años con quemaduras en el año 2020; el lugar donde ocurrió la lesión, por incremento de la proporción de niños quemados dentro del hogar propio $(\mathrm{p} \leq 0,0001)$ y el aumento del porcentaje de niños que viven solo con la madre. $(\mathrm{p} \leq 0,001)$ (tabla 2$)$.

De las características clínicas, hubo un cambio significativo en el agente causal; principalmente se observó un aumento en quemaduras por líquido caliente ( $\mathrm{p} \leq 0,001)$, un aumento en el número de localizaciones por quemaduras ( 3 o más lesiones) $(\mathrm{p} \leq 0,03)$ y el tratamiento inicial otorgado en el lugar de ocurrencia de la lesión, observándose un aumento de únicamente aplicación de agua en el periodo de pandemia $(\mathrm{p} \leq 0,001)($ tabla 3$)$. 
Tabla 1. Pacientes con quemaduras atendidos en COANIQUEM y comuna de Pudahuel según mes y año de estudio

\begin{tabular}{|c|c|c|c|c|c|c|c|c|}
\hline \multirow[t]{2}{*}{ Mes } & \multicolumn{3}{|c|}{ COANIQUEM } & \multirow{2}{*}{$\begin{array}{c}\% \text { de cambio } \\
2017-2019 \text { a } 2020\end{array}$} & \multirow{2}{*}{$\begin{array}{l}\% \text { de cambio } \\
2019-2020\end{array}$} & \multicolumn{2}{|c|}{ Comuna de Pudahuel } & \multirow{2}{*}{$\begin{array}{l}\% \text { de } \\
\text { cambic }\end{array}$} \\
\hline & Promedio 2017-2019 & 2019 & 2020 & & & 2019 & 2020 & \\
\hline Abril & 281 & 310 & 129 & $-54,1$ & $-58,4$ & 23 & 7 & $-69,6$ \\
\hline Mayo & 337 & 328 & 117 & $-65,3$ & $-64,3$ & 27 & 5 & $-81,5$ \\
\hline Junio & 366 & 307 & 217 & $-40,7$ & $-29,3$ & 30 & 23 & $-23,3$ \\
\hline Julio & 384 & 395 & 224 & $-41,7$ & $-43,3$ & 38 & 21 & $-44,7$ \\
\hline Total & 1.357 & 1.340 & 687 & $-49,4$ & $-48,7$ & 118 & 56 & $-52,5$ \\
\hline
\end{tabular}

\section{Discusión}

En el período estudiado del año 2020, se produjo una importante disminución del ingreso de pacientes ambulatorios a COANIQUEM $(48,7 \%)$ en relación al año anterior. Esta disminución podría explicarse, en parte, por la estricta cuarentena de los primeros meses de la pandemia, lo que dificultó el desplazamiento de los pacientes, especialmente desde otras ciudades y también, por el temor de las familias al contagio por SARS CoV-2 al acudir a un centro asistencial y al utilizar el transporte público. Sin embargo, la reducción de ingresos de pacientes de la comuna de Pudahuel registrado en tabla 1, que tuvieron menores dificultades de desplazamiento por vivir cerca del Centro de Rehabilitación, apoyaría el supuesto que se produjo una menor cantidad de quemaduras.

A nivel internacional, los informes son contradictorios; algunos centros reportan un aumento de admisiones y hospitalizaciones como es el caso del Centro de Atención Pediátrica de Turquía, con un 52\% y 60\% respectivamente para la población menor de 18 años entre el 11 de marzo y 11 de junio 2020, atribuible a las medidas sanitarias de "todos quedarse en casa"26, o el Centro Pediátrico de Trauma en Polonia, en un análisis de los meses de enero a marzo de los años 2016 a 2020, registra una diferencia significativa para el mes de marzo 2020 con un aumento de 2,7 veces de los casos hospitalizados por quemaduras. Comentan los autores que el aumento del número de casos de quemaduras pediátricas podría estar relacionado con el hecho de que los niños se quedaban en casa con sus padres, quienes los debían cuidar, pero además teletrabajar en casa $^{27}$.

Otros artículos comparten nuestros hallazgos, como el Centro Pediátrico de Quemaduras en el Reino Unido, en una comparación retrospectiva durante cinco semanas de confinamiento impuestas por el gobierno del 23 de marzo 2020 al 30 de abril 2020 con un periodo similar de 2019, encuentran una disminución del $37 \%$ en los pacientes ambulatorio ${ }^{20}$ o, en el Centro de Atención Pediátrica de Bucarest, Rumania, en un estudio comparativo entre el 16 de marzo y 15 de mayo de 2020 , con el mismo periodo del 2019, se produjo un descenso del 50,8\% del número total de pacientes que llegaron a la sala de emergencias con trauma y quemaduras, y las admisiones de niños con quemaduras disminuyeron en $41 \%{ }^{28}$.

Cabe señalar que en estos reportes internacionales las cifras de atendidos son de poca magnitud y con períodos de observación de 1 a 3 meses ${ }^{13-14,18-21,24,26-30}$. Hay más concordancia en la literatura en que los casos leves y los secundarios a contacto con objetos calientes han disminuido $20,28,30,31$, y que algunos pacientes con quemaduras de mayor gravedad han consultado con retraso ${ }^{29,31}$.

No hay cifras oficiales aún sobre hospitalizaciones de los casos más graves en el país para aseverar que éstas también hayan disminuido a consecuencia de la pandemia. Sin embargo, los pacientes ingresados a COANIQUEM por secuelas que ingresan para iniciar su rehabilitación, disminuyeron en similar proporción que los pacientes que consultan por lesiones recientes en policlínico de curaciones, siendo estos últimos pacientes de menor gravedad por requerir sólo tratamiento ambulatorio. Los ingresos a rehabilitación en cambio corresponden a aquellos que estuvieron hospitalizados, cuya internación no podía postergarse por la mayor profundidad o extensión de su quemadura. Estos resultados podrían estar indicando una menor incidencia en general de quemaduras en NNA lo que inicialmente no se esperaba por los antecedentes epidemiológicos de alta incidencia de quemaduras en el hogar.

En la zona central de Chile, de donde procede el mayor número de nuestros consultantes, las temperaturas descienden progresivamente entre abril y julio al llegar el invierno. ${ }^{32}$ Inversamente, el número de niños con quemaduras aumenta, como se observa en la tabla 1, debido al uso de elementos de calefacción, consumo de líquidos calientes y mayor permanencia de los niños dentro del hogar, lo que facilita el acercamiento a las cocinas.

$\mathrm{Al}$ contrastar los ingresos a COANIQUEM del año 
Tabla 2. Características demográficas y sociales de los pacientes con quemaduras atendidos en CONIQUEM desde abril a julio. Años 2019 y 2020

\begin{tabular}{|c|c|c|c|c|c|}
\hline \multirow{3}{*}{ Característica } & \multicolumn{4}{|c|}{ Año } & \multirow[b]{3}{*}{$p$-value } \\
\hline & \multicolumn{2}{|c|}{2019} & \multicolumn{2}{|c|}{2020} & \\
\hline & $\mathrm{N}^{\circ}$ & $\%$ & $\mathrm{~N}^{\circ}$ & $\%$ & \\
\hline Total & 1340 & 100,0 & 687 & 100,0 & \\
\hline \multicolumn{6}{|l|}{ Género } \\
\hline Femenino & 658 & 50,2 & 331 & 48,2 & \multirow{2}{*}{0,1323} \\
\hline Masculino & 682 & 49,8 & 356 & 51,8 & \\
\hline \multicolumn{6}{|l|}{ Edad } \\
\hline $0-4$ & 791 & 59,0 & 448 & 65,2 & \multirow{4}{*}{0,0263} \\
\hline $5-9$ & 310 & 23,1 & 122 & 17,8 & \\
\hline $10-14$ & 156 & 11,6 & 78 & 11,4 & \\
\hline $15-19$ & 83 & 6,2 & 39 & 5,7 & \\
\hline Promedio \pm DS & \multicolumn{2}{|c|}{$5,93 \pm 4,38$} & \multicolumn{2}{|c|}{$5,08 \pm 4,70$} & \\
\hline \multicolumn{6}{|l|}{ Previsión } \\
\hline Sistema estatal $(\mathrm{A})^{*}$ & 468 & 34,9 & 220 & 32,0 & \multirow{4}{*}{0,2490} \\
\hline Sistema estatal $(B, C \text { y } D)^{* *}$ & 636 & 47,5 & 324 & 47,2 & \\
\hline Sistema privado*** & 183 & 13,7 & 118 & 17,2 & \\
\hline Sin dato & 53 & 4,0 & 25 & 3,6 & \\
\hline \multicolumn{6}{|l|}{ Zona de procedencia } \\
\hline Norte del país & 199 & 14,9 & 108 & 15,7 & \multirow{4}{*}{0,7249} \\
\hline Región Metropolitana & 833 & 62,1 & 436 & 63,5 & \\
\hline Sur del país & 304 & 22,7 & 141 & 20,5 & \\
\hline Extranjero & 4 & 0,3 & 2 & 0,3 & \\
\hline \multicolumn{6}{|l|}{ Centro de atención } \\
\hline Antofagasta & 152 & 11,3 & 90 & 13,1 & \multirow{3}{*}{0,3867} \\
\hline Región Metropolitana & 934 & 69,7 & 460 & 67,0 & \\
\hline Puerto Montt & 254 & 19,0 & 137 & 19,9 & \\
\hline \multicolumn{6}{|l|}{ Donde ocurrió la lesión } \\
\hline Hogar propio & 1060 & 79,1 & 635 & 92,4 & \multirow{7}{*}{$<0,000$} \\
\hline Casa familiar/vecino & 189 & 14,1 & 41 & 6,0 & \\
\hline Vía pública & 23 & 1,7 & 5 & 0,7 & \\
\hline Aire libre & 22 & 1,6 & 3 & 0,4 & \\
\hline Jardin infantil/colegio & 16 & 1,2 & - & & \\
\hline Otro & 11 & 0,8 & - & & \\
\hline Sin dato & 19 & 1,4 & 3 & 0,4 & \\
\hline \multicolumn{6}{|l|}{ Con quién vive } \\
\hline Ambos padres & 863 & 64,4 & 428 & 62,3 & \multirow{6}{*}{0,0009} \\
\hline Solo con la madre & 373 & 27,8 & 229 & 33,3 & \\
\hline Otro pariente & 39 & 2,9 & 9 & 1,3 & \\
\hline Solo con el padre & 9 & 0,7 & 9 & 1,3 & \\
\hline Otra situación & 4 & 0,3 & 2 & 0,3 & \\
\hline Sin dato & 52 & 3,9 & 10 & 1,5 & \\
\hline
\end{tabular}

*FONASA A: Personas carentes de recursos y personas migrantes, causantes de subsidio familiar (Ley 18.020). Bonificación 100\%. **FONASA B: Personas que perciben un ingreso imponible mensual $\leq \$ 319.000$. Bonificación $100 \%$ y compra bono libre elección. 1 DÓLAR $=\$ 788$. $* *$ FONASA C: Personas que perciben un ingreso imponible mensual $\leq \$ 319.000 \mathrm{y}<\$ 465.740$. Bonificación $90 \%$ o compra bono libre elección. ${ }^{*}$ FONASA D: Personas que perciben un ingreso imponible mensual $\geq \$ 465.740$. Bonificación $\$ 80 \%$ o compra bono libre elección. *** Incluye seguros privados y de Fuerzas Armadas 
Tabla 3. Características clínicas de los pacientes con quemaduras atendidos en COANIQUEM desde abril a julio. Años 2019 y 2020

\begin{tabular}{|c|c|c|c|c|c|}
\hline \multirow{3}{*}{ Característica } & \multicolumn{4}{|c|}{ Año } & \multirow{3}{*}{$p$-value } \\
\hline & \multirow{2}{*}{$\begin{array}{c}2019 \\
\text { Frecuencia }\end{array}$} & \multicolumn{3}{|c|}{2020} & \\
\hline & & $\%$ & Frecuencia & $\%$ & \\
\hline Total & 1340 & 100,0 & 687 & 100,0 & \\
\hline \multicolumn{6}{|l|}{ Tipo de ingreso } \\
\hline Agudo & 1179 & 88,0 & 611 & 88,9 & \multirow[t]{2}{*}{0,2208} \\
\hline Secuela & 161 & 12,0 & 76 & 11,1 & \\
\hline \multicolumn{6}{|l|}{ Agente } \\
\hline Líquido caliente & 695 & 51,9 & 422 & 61,4 & \multirow[t]{7}{*}{0,0012} \\
\hline Objeto caliente & 566 & 42,2 & 224 & 32,6 & \\
\hline Fuego y brasas & 39 & 2,9 & 18 & 2,6 & \\
\hline Fricción & 11 & 0,8 & 5 & 0,7 & \\
\hline Electricidad & 9 & 0,7 & 10 & 1,5 & \\
\hline Sustancia química/frio & 11 & 0,8 & 4 & 0,6 & \\
\hline Desconocido & 9 & 0,7 & 4 & 0,6 & \\
\hline \multicolumn{6}{|l|}{ Profundidad } \\
\hline Superficial & 279 & 20,8 & 143 & 20,8 & \multirow[t]{4}{*}{0,0846} \\
\hline Intermedia & 929 & 69,3 & 494 & 71,9 & \\
\hline Profunda & 108 & 8,1 & 46 & 6,7 & \\
\hline Sin datos & 24 & 1,8 & 4 & 0,6 & \\
\hline \multicolumn{6}{|l|}{ No localizaciones } \\
\hline 1 & 865 & 64,6 & 414 & 60,3 & \multirow[t]{4}{*}{0,0374} \\
\hline 2 & 272 & 20,3 & 135 & 19,7 & \\
\hline $3-5$ & 173 & 12,9 & 114 & 16,6 & \\
\hline $6-10$ & 30 & 2,2 & 24 & 3,5 & \\
\hline \multicolumn{6}{|l|}{ Hora de lesión } \\
\hline $0-8$ & 92 & 6,9 & 33 & 4,8 & \multirow[t]{7}{*}{0,2382} \\
\hline $9-12$ & 226 & 16,9 & 121 & 17,6 & \\
\hline $13-16$ & 245 & 18,3 & 149 & 21,7 & \\
\hline $17-20$ & 457 & 34,1 & 218 & 31,7 & \\
\hline $21-24$ & 295 & 22,0 & 153 & 22,3 & \\
\hline Sin datos & 25 & 1,9 & 13 & 1,9 & \\
\hline Promedio \pm DS & \multicolumn{2}{|c|}{$16,358 \pm 5,218$} & \multicolumn{2}{|c|}{$16,498 \pm 5,903$} & \\
\hline \multicolumn{6}{|l|}{ Localización } \\
\hline Mano & 522 & 39,0 & 241 & 35,1 & \multirow[t]{9}{*}{0,3614} \\
\hline Extremidad superior* & 237 & 17,7 & 122 & 17,8 & \\
\hline Extremidad inferior** & 217 & 16,2 & 113 & 16,4 & \\
\hline Tórax/abdomen & 159 & 11,9 & 81 & 11,8 & \\
\hline Cara/cuello & 109 & 8,1 & 73 & 10,6 & \\
\hline Pie & 63 & 4,7 & 43 & 6,3 & \\
\hline Zona periano-genital & 21 & 1,6 & 8 & 1,2 & \\
\hline Cuero cabelludo & 10 & 0,7 & 6 & 0,9 & \\
\hline Sin datos & 2 & 0,1 & - & - & \\
\hline \multicolumn{6}{|c|}{ Tratamiento en lugar de ocurrencia } \\
\hline Solo agua & 912 & 68,1 & 495 & 72,1 & \multirow[t]{7}{*}{0,0006} \\
\hline Nada & 139 & 10,4 & 60 & 8,7 & \\
\hline Agua + aditamentos & 127 & 9,5 & 82 & 11,9 & \\
\hline Otros & 69 & 5,1 & 27 & 3,9 & \\
\hline Agua+hielo+aditamentos & 56 & 4,2 & 12 & 1,7 & \\
\hline Aceite+aditamentos & 21 & 1,6 & 3 & 0,4 & \\
\hline Desconocido & 16 & 1,2 & 8 & 1,2 & \\
\hline
\end{tabular}

* No incluye mano. ${ }^{* *}$ No incluye pie 
2020 con los del año 2019, se observa una disminución considerable de pacientes. Sin embargo, al comparar el mes de junio entre estos dos años, la disminución de los ingresos no sigue la misma tendencia que los otros meses. Esto se considera algo puntual al comparar el promedio de ingresos de los tres años anteriores, descartando así que algún factor climático afecte dicha comparación. Es más, se observa de forma más clara la tendencia de aumento de casos entre abril y julio al observar el promedio de ingresos de los meses entre el año 2017 y 2019 con totales promedios similares a 2019.

No hubo diferencia significativa en ambos períodos en cuanto al género, pero sí en edad. El aumento relativo de un $10,5 \%$ en los menores de 5 años con quemaduras en 2020 podría corresponder a que los niños de esa edad habitualmente son los que más se queman dentro del hogar, lugar donde han tenido que permanecer más tiempo por las restricciones.

La menor demanda de atención afectó por igual a los tres centros, sin notarse efecto debido a la residencia en las diferentes zonas geográficas del país. El menor nivel socioeconómico, tampoco provocó una diferencia en la menor incidencia del año 2020, en relación a los otros grupos.

En el año 2020 disminuyeron en un 16,8\% las quemaduras fuera del hogar propio, pues al estar los padres también confinados, sin ir a sus lugares de trabajo, no enviaron a sus hijos donde otros familiares o vecinos. Así mismo, fue mayor el riesgo de quemarse en hogares monoparentales constituidos sólo por la madre, secundario a la sobre-ocupación de la mujer y las menores posibilidades de supervisión a los niños, lo que probablemente sí se logra en salas-cuna y jardines infantiles y que, por la pandemia, no funcionaron de manera presencial.

El aumento relativo de ingresos por agentes líquidos calientes $(18,3 \%)$ se podría explicar por la disminución simultánea de quemaduras por objetos calientes. Esto último podría estar influido por un menor uso de elementos de calefacción en abril y mayo 2020 por las temperaturas más templadas durante ese año y porque los objetos calientes provocan lesiones de menor magnitud, motivo por el que los padres podrían decidir no consultar y efectuar curaciones caseras.

El aumento de lesiones múltiples con 3 o más localizaciones por paciente $(33,1 \%)$ se debería al mayor porcentaje relativo en 2020 de quemaduras por líquidos calientes. La disminución de quemaduras en manos también estaría relacionada a una menor exposición a objetos calientes, aunque no alcanzó significancia estadística.

Un hallazgo interesante fue el mejor manejo inicial de las quemaduras a expensas de utilizar agua sola o combinada, lo que indicaría una favorable difusión de consejos preventivos entre los padres, en este caso como prevención secundaria. Sin embargo, se carece de evidencia suficiente para afirmarlo, puesto que, no hay estudios referidos al período analizado sobre el impacto de la campaña comunicacional en redes sociales efectuada por COANIQUEM. En todo caso, nuestros resultados indican una disminución de los consultantes con quemaduras, lo que ya sería positivo respecto de la hipótesis inicial.

Durante los meses estudiados se produjo el mayor incremento del número mensual de contagiados en el país por el SARS CoV2 durante el año 2020 alcanzando su punto máximo en junio.

La curva de este fenómeno epidemiológico no se correlaciona en forma significativa con la evolución de la demanda de atención de los pacientes con quemaduras, lo que podría indicar que el respeto a las medidas de confinamiento de la población se mantuvo de manera uniforme en todo el período debido al temor de contagio.

Como limitaciones al estudio, se puede mencionar la duración de éste, que se remite al período inicial y más crítico de la pandemia en el país. Se hace necesario una revisión de un período más prolongado para observar lo que pudo haber pasado con pacientes que no consultaron teniendo lesiones que les hayan provocado secuelas de menor consideración y que podrían solicitar consulta en un período más alejado del episodio inicial, al mejorar las condiciones de desplazamiento desde localidades distantes de los establecimientos. Esta situación también ha afectado las publicaciones que se han efectuado sobre este tema, varias de las cuales corresponden a cartas al editor y se refieren en su gran mayoría al período entre marzo y junio del 2020.

\section{Conclusiones}

En el período inicial de la pandemia por SARS $\mathrm{CoV} 2$, con confinamiento estricto, se produjo una disminución de la demanda de atención ambulatoria por quemaduras en COANIQUEM, contraria a lo esperado, afectando por igual a pacientes ambulatorios con lesiones agudas y pacientes con requerimiento de rehabilitación de secuelas, secundario a la menor posibilidad de recurrir a centros de atención de salud y a una disminución efectiva de la ocurrencia de quemaduras.

\section{Responsabilidades Éticas}

Protección de personas y animales: Los autores declaran que los procedimientos seguidos se conformaron a las normas éticas del comité de experimentación humana responsable y de acuerdo con la Asociación Médica Mundial y la Declaración de Helsinki. 
Confidencialidad de los datos: Los autores declaran que han seguido los protocolos de su centro de trabajo sobre la publicación de datos de pacientes.

Derecho a la Privacidad y Consentimiento Informado: Este estudio ha sido aprobado por el Comité de Ética de Investigación correspondiente, quien de acuerdo a las características del estudio ha eximido el uso del Consentimiento Informado.

\section{Conflicto de intereses}

Los autores declaran no tener conflicto de intereses.

\section{Referencias}

1. Veloso L. Ministerio de Salud confirma primer caso de Coronavirus en Chile. 2020. [Documento en línea]. Disponible en: https://n9.cl/6mqn [Consultado el 2020-7-19].

2. Romero M, Vallejos L. Informe epidemiológico: Las 100 comunas que no registran casos confirmados de coronavirus. 2020. [Documento en línea]. Disponible en: https://n9.cl/47tc1 [Consultado el 2020-07-19].

3. BBC News Mundo. Coronavirus: Chile decreta el "estado de catástrofe" en todo el país por 90 días ante el avance de la enfermedad. 2020. [Documento en línea]. Disponible en: https://n9.cl/4xta [Consultado el 2020-07-19].

4. Romero M. Gobierno amplía estado de excepción constitucional de catástrofe por 90 días en medio de la pandemia. 2020. [Documento en línea]. Disponible en: https://n9.cl/izng2. [Consultado el 202007-19].

5. Cooperativa.cl. Piñera ordenó toque de queda en todo Chile entre las 22:00 y las 05:00 horas. 2020. [Documento en línea]. Disponible en: https://n9.cl/4xki8. [Consultado el 2020-07-19].

6. Blanco M. Toque de queda en Chile: ¿quiénes podrán acceder a los salvoconductos para circular en todo el país? 2020. [Documento en línea]. Disponible en: https://n9.cl/itfhp. [Consultado el 2020-07-19].

7. Veloso L. Mineduc informa suspensión de clases presenciales hasta que condiciones sanitarias permitan retorno. 2020. [Documento en línea]. Disponible en: https://n9.cl/biobio23abril. [Consultado el 2020-07-19].

8. T13. Vacaciones de inverno serán en abril por coronavirus. 2020. [Documento en línea]. Disponible en: https://n9.cl/7xlko. [Consultado el 2020-07-19].

9. Meganoticias. Vacaciones de invierno adelantadas: ¿Cuándo finalizan según el calendario de Mineduc? 2020. [Documento en línea]. Disponible en: https://n9.cl/aushx. [Consultado el 202007-19].
10. Data UC. Visualizador Covid-19 Chile. 2020. [Documento en línea]. Disponible en: https://coronavirus.mat.uc.cl/. [Consultado en 2020-12-7].

11. Rojas MA, Saavedra R, Vicencio P, et al. Cambios epidemiológicos en niños quemados a 10 años de seguimiento. Rev Chil Pediatr. 2016;87:186-92.

12. Solís F, Domic C, Saavedra R. Epidemiología de las quemaduras en niños y adolescentes de Región Metropolitana de Chile. Rev Chil Pediatr 2014;85:690-700.

13. Barret J, Chong S, Depetris N, et al. Burn center function during the COVID-19 pandemic: An international multi-center report of strategy and experience. Burns 2020;46:1021-35.

14. Toh V, Antrum J, Sloan B, et al. Management of Covid-19 in burns patients: The experience of a UK burn centre. Burns 2020;46:1707-28.

15. Li N, Liu T, Cheng H, et al. Management strategies for the burn ward during COVID-19 pandemic. Burns 2020;46:75661.

16. Nischwitz S, Popp D, Sawetz I, et al. Burns in pandemic times - The Graz way towards COVID-19 and back. Burns 2021;47:234-9.

17. Ma S, Yuan Z, Peng Y, et al. Experience and suggestion of medical practices for burns during the outbreak of COVID-19. Burns 2020;46:749-55.

18. Chu H, Reid G, Sack A, et al. Changes in burn referrals and injuries during CoVid-19. Burns 2020;46:1469-70.

19. Soto C, Macchiavello R, Alarcón J, et al. Early experience with severely burned COVID-19 patients at the National Reference Center for adult major burns in Chile. Burns (en prensa) 2020, https://doi.org/10.1016/j. burns.2020.04.041.

20. D'Asta F, Choong J, Thomas C, et al. Paediatric burns epidemiology during COVID-19 pandemic and 'stay home' era. Burns 2020;46:1471-2.

21. Farroha A. Effects of COVID-19 pandemic on burns epidemiology. Burns 2020;46:1466.

22. Ryan C, Stoddard F, Kazis L, et al.
COVID-19 pandemic and the burn survivor community: A call for action. Burns 2021;47(1):250-1.

23. Kamolz L, Schiefer J, Horter J, et al. COVID-19 and burns: Lessons learned? Burns 2020;46:1467-8.

24. Farroha A. Reduction in length of stay of patients admitted to a regional burn centre during COVID-19 pandemic. Burns 2020;46:1715.

25. Gob.cl. [Archivo] El Ministerio de Salud junto a COANIQUEM lanzan Campaña "Alto al Fuego". [Documento en línea]. Disponible en: https://n9.cl/ovy4s. [Consultado 2021-03-25].

26. Demircan M. Increased admissions and hospitalizations to pediatric burn center during COVID 19 pandemic. Burns 2021;47:479-501.

27. Kawalec A. The changes in the number of patients admissions due to burns in Paediatric Trauma Centre in Wroclaw (Poland) in March 2020. Burns 2020;46(7):1713-4.

28. Tatar R, Enescu D. The impact of COVID-19 pandemic on the activity of a pediatric burn center in Bucharest, Romania, Burns 2020;46:1977-8.

29. Phillips G, Talwar C, Makaranka S, et al. The impact and lessons learnt from the COVID-19 pandemic in a UK Burns Centre. Burns (en prensa) 2021; https:// doi.org/10.1016/j.burns.2021.01.008.

30. Akkoç M, Bülbüloğlu S, Özdemir M. The effects of lockdown measures due to COVID-19 pandemic on burn cases. Int Wound J. 2020;1-8.

31. Yaacobi D, Ad-El D, Kalish E, et al. Management Strategies for Pediatric Burns During the COVID-19 Pandemic. Journal of Burn Care \& Research 2021;42(2):141-3.

32. Meteored. Datos históricos del tiempo en Santiago de Chile. Resumen de datos mensuales para abril, mayo, junio y julio de 2019 y 2020. [Documento en línea]. Disponible en: https:// www.meteored.cl/tiempo-en_ Santiago+de+Chile-America+Sur-ChileRegion+Metropolitana+de+SantiagoSCEL-sactual-18578.html. [Consultado 2020-12-7]. 